\title{
Pleiotropic Effects of Long-Term Monotherapy with Rosuvastatin in Dogs with Moderate Heart Failure
}

\author{
Valerio Zacà Sudhish Mishra Ramesh C. Gupta Sharad Rastogi \\ Hani N. Sabbah \\ Division of Cardiovascular Medicine, Department of Medicine, Henry Ford Hospital, Detroit, Mich., USA
}

\section{Key Words}

Heart failure $\cdot$ Left ventricular myocardium $\cdot$ Rosuvastatin
Conclusion: These pleiotropic effects of RSV may account, in part, for the observed beneficial effect of RSV on LV function and structural remodeling.

Copyright $\odot 2012$ S. Karger AG, Basel

\begin{abstract}
Objective: The objective of this study was to investigate the potential pleiotropic effects of rosuvastatin (RSV) in the left ventricular (LV) myocardium of dogs with moderate heart failure (HF). Methods: LV tissue was obtained from HF dogs randomized to 3 months therapy with low-dose RSV $(n=7)$, high-dose RSV ( $n=7$ ) or to no therapy (Control, $n=7$ ) and from 7 normal dogs. mRNA and protein expression of prohypertrophic mediator NGFI-A binding protein 1 (Nab1), phosphatase and tensin homolog (PTEN), phosphoinositide-3 kinase (PI3K) and mammalian target of rapamycin (mTOR) were measured, as well as that of proinflammatory cytokine interleukin-6 (IL-6), bone marrow-derived stem cell markers cKit and Sca1, vascular endothelial and fibroblast growth factors (VEGF and FGF) and nitric oxide synthase (NOS) isoforms. Results: Nab1, PTEN, PI3K, mTOR and IL-6 increased in the controls. High-dose RSV reduced expression of Nab1, PTEN, PI3K, mTOR and IL- 6 to near-normal levels. cKit and Sca1 significantly increased, while VEGF and FGF decreased in the controls compared to the normal dogs. RSV therapy further increased expression of cKit, Sca1, VEGF and FGF. High-dose RSV normalized the expression of NOS isoforms.
\end{abstract}

\section{Introduction}

The 3-hydroxy-3-methylglutaryl co-enzyme A reductase inhibitors, or statins, have been consistently shown to reduce morbidity and mortality from atherosclerotic cardiovascular disease [1]. Beyond their lipid-lowering action, statins possess pleiotropic effects [2] including anti-inflammatory and antihypertrophic [3], proangiogenic [4] and bone marrow-derived stem cell (BMSC) mobilizing properties $[3,5,6]$, potentially providing a biological rationale for their use in the prevention and treatment of left ventricular (LV) dysfunction and heart failure (HF). Overall, albeit partially disappointing $[7,8]$, the gathered evidence on the use of statins in HF due to LV systolic dysfunction suggests that their use is safe and is associated with improved LV ejection fraction (EF) and decreased hospitalization for worsening HF [9]. Recently, encouraging data have been reported with higher statin doses [10].

\section{KARGER}

Fax +4161306 1234

E-Mail karger@karger.ch

www.karger.com (c) 2012 S. Karger AG, Basel

$0008-6312 / 12 / 1233-0160 \$ 38.00 / 0$

Accessible online at:

www.karger.com/crd
Hani N. Sabbah, $\mathrm{PhD}$

Director, Cardiovascular Research, Henry Ford Hospital

2799 West Grand Boulevard

Detroit, MI 48202 (USA)

E-Mail hsabbah1@hfhs.org 
We showed previously that early, long-term monotherapy with high-dose (HD) rosuvastatin (RSV) prevents the progressive LV dysfunction and remodeling seen in dogs with moderate HF [3]. These beneficial effects on LV function and global cardiac remodeling are associated at the cellular level with reduced myocardial fibrosis and myocyte hypertrophy, and with increased capillary density. HD RSV also showed anti-inflammatory properties by downregulating TNF- $\alpha$ and gelatinase expression, and stimulated the mobilization of circulating Scal-positive BMSCs. Similar findings have been partly confirmed in patients with chronic HF [10], yet the underlying mechanisms and the pathways involved in mediating such positive effects are only partially known.

In this study, we examined the effects - in the LV tissue of dogs with moderate HF - of RSV monotherapy on the mRNA and protein expression of specific prohypertrophic mediators and proinflammatory cytokines and of angiogenetic growth factors, BMSC markers and nitric oxide synthase (NOS) isoforms. In all of these, pleiotropic properties potentially account for the observed beneficial effects of HD RSV in this model of HF.

\section{Methods}

\section{Experimental Model}

The canine model of chronic postischemic HF used in this study was previously described in detail [11]. In this preparation, $\mathrm{LV}$ dysfunction is produced by multiple sequential coronary microembolizations with polystyrene latex microspheres (70-102 $\mu \mathrm{m}$ in diameter), which results in loss of viable myocardium. The model presents many of the hemodynamic and neurohormonal sequelae of HF observed in humans, including marked and progressive depression of LV systolic and diastolic function, reduced cardiac output and increased LV filling pressures. In our study, 21 healthy, normocholesterolemic mongrel dogs, weighing between 20 and $30 \mathrm{~kg}$, underwent serial coronary microembolizations to produce HF. To create moderate HF, coronary microembolizations were performed 1-3 weeks apart and were discontinued when the LV EF, determined angiographically, was between 30 and $40 \%$. All the procedures were performed during cardiac catheterization under general anesthesia and sterile conditions. The anesthesia regimen consisted of a combination of intravenous administration of oxymorphone $(0.22 \mathrm{mg} / \mathrm{kg})$, diazepam $(0.17 \mathrm{mg} /$ $\mathrm{kg}$ ) and sodium pentobarbital (150-250 $\mathrm{mg}$ to effect). The study was approved by the Henry Ford Hospital Care of Experimental Animals Committee and conformed to the 'Position of the American Heart Association on Research Animal Use' [12].

Study Protocol

Two weeks after the last embolization, the dogs underwent a prerandomization left and right cardiac catheterization. One day later, they were randomized to 3 months of oral monotherapy with low-dose (LD) RSV (0.5 mg once daily, $\mathrm{n}=7$ ), HD RSV (3.0 $\mathrm{mg}$ once daily, $\mathrm{n}=7$ ) or no therapy at all (Control, $\mathrm{n}=7$ ). At the end of the follow-up period, a final left and right cardiac catheterization was performed, then under general anesthesia the chest was opened and the heart rapidly removed for subsequent histological and biochemical examination. LV tissue samples were likewise obtained and processed from 6 normal dogs for comparisons. The results of the hemodynamic, angiographic and histomorphometric analyses performed on the dogs enrolled in the study were published previously [3].

\section{$m R N A$ and Protein Expression}

All tissue samples were submitted for analysis without treatment regimen identifiers. mRNA expression of glyceraldehyde-3phosphate dehydrogenase (GAPDH), prohypertrophic mediator NGFI-A binding protein 1 (Nab1), phosphatase and tensin homolog (PTEN), phosphoinositide-3 kinase (PI3K) and mammalian target of rapamycin (mTOR) were measured, as well as that of the proinflammatory cytokines interleukin-6 (IL-6), BMSC markers cKit and Scal [3], vascular endothelial and fibroblast growth factors (VEGF and FGF) and endothelial, inducible and neuronal NOS (eNOS, iNOS and nNOS) isoforms. Total RNA with an absorbance ratio $(260 \mathrm{~nm} / 280 \mathrm{~nm}$ ) above 1.7 was isolated from frozen LV tissue and approximately 4-10 $\mu$ g RNA was reverse-transcribed into cDNA in an assay volume of $80 \mu \mathrm{l}$ as described previously $[13,14]$. Protein levels of Nab1, PTEN, PI3K, mTOR, IL-6, cKit, Sca1, VEGF, FGF, eNOS, iNOS and nNOS were measured in LV homogenate by Western blots as described previously [13, 14]. Primary antibodies specific to each protein were diluted based on the supplier's instructions (Santa Cruz Biotechnology Inc., Santa Cruz and Chemicon, Temecula, Calif., Cell Signalling, Danvers, Mass. and BD Biosciences). In all instances, the antibody was present in excess over the antigen and the density of each protein band was in the linear scale. Band intensities were quantified in densitometric units.

\section{Statistical Analysis}

In the original study [3], a power calculation based on LV EF as being the primary end point of interest, a sample size of $n=7$ per study group was sufficient to provide $80 \%$ power to detect effect sizes of 1.52 standard deviations or more by Student $t$ test. In this study, between-groups comparisons were performed by using a 1-way analysis of variance (ANOVA), with $\alpha$ set at 0.05 . If significance was attained, pairwise comparisons were performed by means of the Newman-Keuls test, with $p<0.05$ considered significant. All the data are reported as the mean \pm SEM.

\section{Results}

The results of the hemodynamic, angiographic and histomorphometric analyses performed on the dogs enrolled in the study have been previously reported [3]. All dogs were normocholesterolemic and no significant differences in total cholesterol and tryglicerides levels were observed at any of the study time points [3]. 
Fig. 1. Bands depicting mRNA expression. From left to right: bands 1 and 2 are from normal dogs, bands 3 and 4 are from untreated HF dogs, bands 5 and 6 are from $\mathrm{HF}$ dogs treated with LD RSV and bands 7 and 8 are from HF dogs treated with HD RSV. Nab1 = NGFI-A binding protein; PTEN = phosphatase and tensin homolog; PI3K = phosphoinositide-3 kinase; $\mathrm{mTOR}=$ mammalian target or rapamycin; IL-6 = interleukin-6; ckit and Scal = bone marrow derived stem cell markers; VEGF $=$ vascular endothelial growth factor; $\mathrm{bFGF}=$ Basic fibroblast growth factor; eNOS = endothelial nitric oxide synthase; iNOS = inducible nitric oxide synthase; $\mathrm{nNOS}=$ neuronal nitric oxide synthase; GAPDH = glyceraldehyde 3-phosphate dehydrogenase.

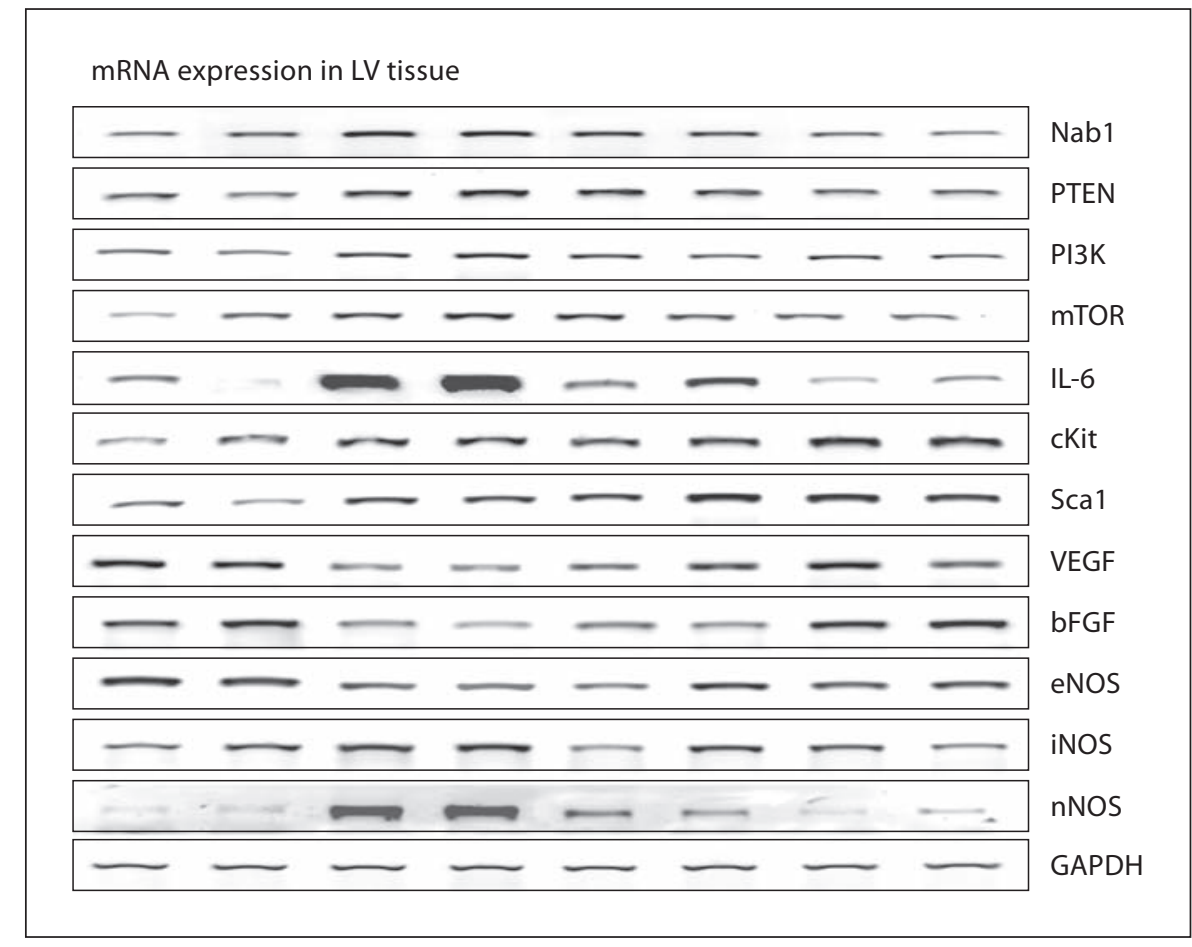

Table 1. mRNA expression of all studied genes in densitometric units

\begin{tabular}{|c|c|c|c|c|}
\hline & $\begin{array}{l}\text { Normal } \\
(n=6)\end{array}$ & $\begin{array}{l}\text { HF controls } \\
(\mathrm{n}=7)\end{array}$ & $\begin{array}{l}\text { LD RSV } \\
(\mathrm{n}=7)\end{array}$ & $\begin{array}{l}\text { HD RSV } \\
(\mathrm{n}=7)\end{array}$ \\
\hline Nab1 & $6,607 \pm 485$ & $9,388 \pm 450^{*}$ & $8,396 \pm 769$ & $7,579 \pm 940$ \\
\hline PTEN & $8,024 \pm 332$ & $11,531 \pm 493^{*}$ & $12,109 \pm 334^{*}$ & $9,442 \pm 797^{\dagger, 末 ~}$ \\
\hline PI3K & $6,336 \pm 713$ & $9,994 \pm 480^{*}$ & $9,363 \pm 703^{*}$ & $7,830 \pm 677$ \\
\hline mTOR & $6,146 \pm 850$ & $9,181 \pm 663$ & $8,630 \pm 1,099$ & $6,710 \pm 778$ \\
\hline IL-6 & $7,102 \pm 790$ & $15,611 \pm 2,749^{*}$ & $11,717 \pm 1,865$ & $6,490 \pm 1,090^{\dagger}$ \\
\hline cKit & $624 \pm 38$ & $911 \pm 48^{*}$ & $960 \pm 43^{*}$ & $1,213 \pm 86^{*, \dagger}$ \\
\hline Scal & $822 \pm 80$ & $1,104 \pm 55^{*}$ & $1,220 \pm 71^{*}$ & $1,267 \pm 52^{*}$ \\
\hline VEGF & $10,558 \pm 924$ & $7,178 \pm 270^{*}$ & $11,874 \pm 617^{\dagger}$ & $10,317 \pm 1,128^{\dagger}$ \\
\hline FGF & $3,914 \pm 413$ & $1,926 \pm 391^{*}$ & $1,748 \pm 684^{*}$ & $3,740 \pm 498^{\dagger \neq}$ \\
\hline eNOS & $1,347 \pm 95$ & $876 \pm 66^{*}$ & $1,210 \pm 95^{\dagger}$ & $1,069 \pm 40^{*}$ \\
\hline iNOS & $1,423 \pm 129$ & $1,730 \pm 71$ & $1,511 \pm 115$ & $1,163 \pm 97^{\dagger}$ \\
\hline nNOS & $8,381 \pm 837$ & $16,625 \pm 2,101^{*}$ & $11,522 \pm 1,534^{\dagger}$ & $8,011 \pm 1,020^{\dagger}$ \\
\hline GADPH & $2,571 \pm 109$ & $2,637 \pm 86$ & $2,851 \pm 133$ & $2,655 \pm 111$ \\
\hline
\end{tabular}

Abbreviations as in fig. $1 .{ }^{*} \mathrm{p}<0.05$ vs. normal; ${ }^{\dagger} \mathrm{p}<0.05$ vs. HF controls; ${ }^{\ddagger} \mathrm{p}<0.05$ vs. LD RSV.

Effect of RSV on Prohypertrophic Mediators and Proinflammatory Cytokines

Results of mRNA expression of all selected genes and GADPH are shown in table 1 and in figure 1 . The mRNA expression of Nab1, PTEN, PI3K and mTOR was in- creased in the control HF dogs compared to the normal dogs. Both doses of RSV reduced the levels of Nab1, mTOR, PTEN and PI3K, but the change reached significance only for PTEN with HD RSV. mRNA expression of IL-6 was significantly upregulated in the controls. LD 
Fig. 2. Bands depicting protein expression. From left to right: bands 1 and 2 are from normal dogs, bands 3 and 4 are from untreated HF dogs, bands 5 and 6 are from HF dogs treated with LD RSV and bands 7 and 8 are from HF dogs treated with HD RSV. Abbreviations as in fig. 1.

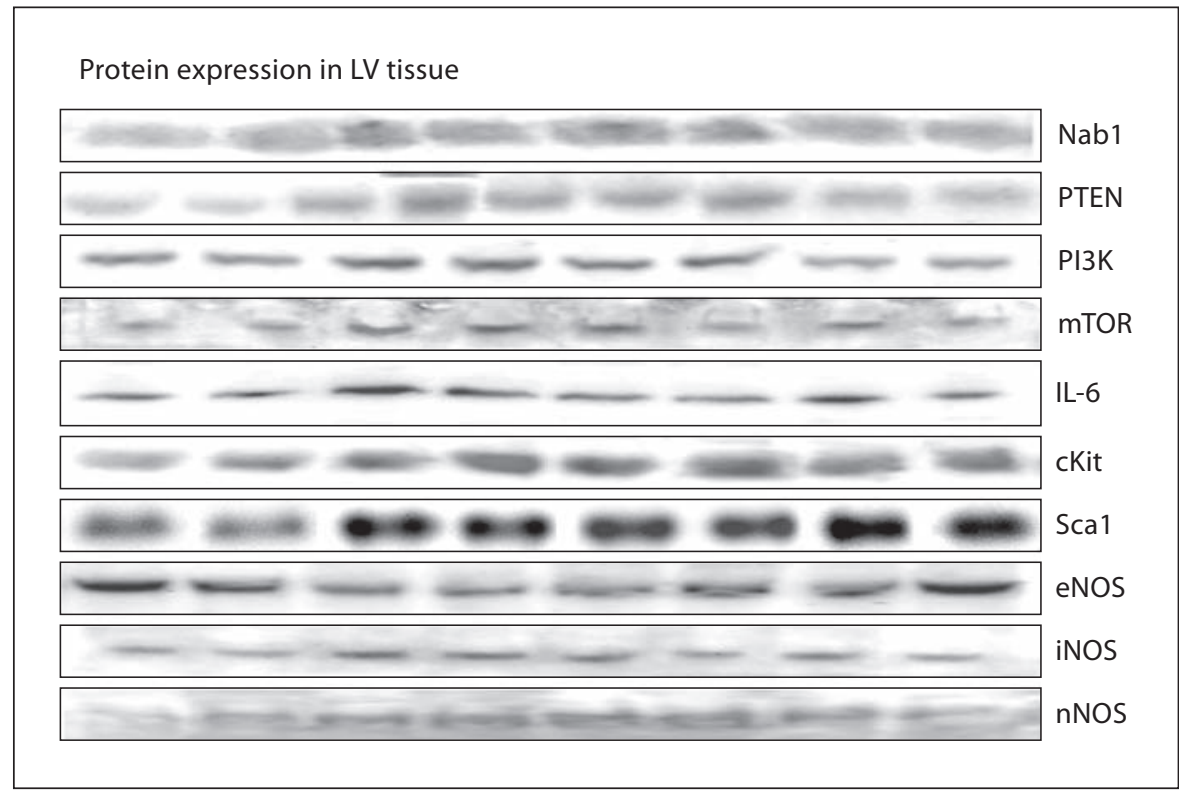

Table 2. Protein expression in densitometric units of prohypertrophic and proinflammatory mediators

\begin{tabular}{lcccc}
\hline & $\begin{array}{l}\text { Normal } \\
(\mathrm{n}=6)\end{array}$ & $\begin{array}{l}\text { HF controls } \\
(\mathrm{n}=7)\end{array}$ & $\begin{array}{l}\text { LD RSV } \\
(\mathrm{n}=7)\end{array}$ & $\begin{array}{l}\text { HD RSV } \\
(\mathrm{n}=7)\end{array}$ \\
\hline Nab1 & $2,521 \pm 40$ & $3,154 \pm 116^{*}$ & $3,368 \pm 148^{*}$ & $2,807 \pm 59^{\dagger, \neq}$ \\
PTEN & $896 \pm 101$ & $1,692 \pm 104^{*}$ & $1,350 \pm 43^{* \dagger}$ & $1,074 \pm 54^{\dagger, \neq}$ \\
PI3K & $2,474 \pm 94$ & $3,289 \pm 59^{*}$ & $2,722 \pm 85^{*} \dagger$ & $2,284 \pm 61^{\dagger, \neq}$ \\
mTOR & $12,276 \pm 434$ & $16,414 \pm 751^{*}$ & $11,731 \pm 715^{\dagger}$ & $10,827 \pm 662^{\dagger}$ \\
IL-6 & $206 \pm 4$ & $307 \pm 20^{*}$ & $222 \pm 9^{\dagger}$ & $227 \pm 10^{\dagger}$ \\
cKit & $2,562 \pm 153$ & $3,424 \pm 93^{*}$ & $3,918 \pm 162^{*}$ & $3,385 \pm 82^{*}$ \\
Scal & $2,179 \pm 259$ & $3,107 \pm 232^{*}$ & $3,579 \pm 99^{*}$ & $3,467 \pm 308^{*}$ \\
\hline
\end{tabular}

Abbreviations as in fig. $1 .{ }^{*} \mathrm{p}<0.05$ vs. normal; ${ }^{\dagger} \mathrm{p}<0.05$ vs. HF controls; ${ }^{\ddagger} \mathrm{p}<0.05$ vs. LD RSV.

RSV therapy was associated with a modest but insignificant reduction of IL-6 levels, while HD RSV therapy normalized mRNA expression of IL- 6 .

Results of protein expression of prohypertrophic and proinflammatory mediators are shown in table 2 and in figure 2. The protein expression of Nab1, PTEN, PI3K and mTOR was significantly increased in the controls compared to the normal dogs. Therapy with both doses of RSV significantly decreased levels of PTEN, PI3K and mTOR compared to the controls, with a more significant reduction seen in the HD RSV-treated dogs. Conversely, only HD RSV treatment was associated with a significant reduction in Nab1 levels which were restored to near-nor- mal values. IL- 6 expression was upregulated in the controls compared to the normal dogs.

\section{Effect of RSV on Growth Factors and Stem Cell Markers}

Table 1 and figure 1 summarize the results of the mRNA expression of VEGF, FGF, cKit and Scal. VEGF and FGF mRNA expression was significantly reduced, while that of cKit and Scal significantly increased in the controls compared to the normal dogs. LD RSV and HD RSV significantly increased the mRNA expression of VEGF with near-normal levels being restored. Only HD RSV was associated with the normalization of FGF expression. mRNA 
Table 3. Protein expression in densitometric units of NO synthase isoforms

\begin{tabular}{lllll}
\hline & $\begin{array}{l}\text { Normal } \\
(\mathrm{n}=6)\end{array}$ & $\begin{array}{l}\text { HF controls } \\
(\mathrm{n}=7)\end{array}$ & $\begin{array}{l}\text { LD RSV } \\
(\mathrm{n}=7)\end{array}$ & $\begin{array}{l}\text { HD RSV } \\
(\mathrm{n}=7)\end{array}$ \\
\hline eNOS & $558 \pm 48$ & $336 \pm 33^{*}$ & $400 \pm 29^{*}$ & $518 \pm 53^{\dagger}$ \\
iNOS & $536 \pm 37$ & $915 \pm 89^{*}$ & $1,023 \pm 36^{*}$ & $739 \pm 67^{*, \neq}$ \\
nNOS & $149 \pm 6$ & $235 \pm 12^{*}$ & $196 \pm 22$ & $194 \pm 17$ \\
\hline
\end{tabular}

${ }^{*} \mathrm{p}<0.05$ vs. normal; ${ }^{\dagger} \mathrm{p}<0.05$ vs. HF controls; ${ }^{*} \mathrm{p}<0.05$ vs. LD RSV.

expression of both cKit and Scal increased significantly with both LD and HD RSV when compared to normal dogs. The ckit levels were further increased significantly with HD RSV (as compared to controls).

The effects of RSV therapy on the protein expression of VEGF and FGF, and of cKit and Scal are shown in figure 3 and in table 2, respectively. The expression of VEGF and FGF was significantly downregulated in the controls compared to the normal dogs. Three months of RSV therapy restored VEGF and FGF expression to near-normal levels. Protein expression of cKit and Scal was significantly enhanced in the controls, and further increases (compared to with the normal dogs) were attained after long-term treatment with both LD RSV and HD RSV.

\section{Effect of RSV on NOS Isoforms}

Results of the mRNA expression of NOS isoforms are shown in table 1 and in figure 1. mRNA expression of eNOS decreased and that of nNOS increased significantly in the controls compared to the normal dogs. mRNA expression of iNOS tended to increase in the controls, but this did not reach statistical significance. LD RSV restored the expression of eNOS to normal levels and reduced, albeit to a lesser extent than HD RSV, both iNOS and nNOS. HD RSV therapy significantly decreased the mRNA expression of nNOS and iNOS and tended to increase the expression of eNOS, but not significantly.

Results of the protein levels of NOS isoforms are shown in table 3 and in figure 2. Protein levels of eNOS decreased significantly, and those of iNOS and nNOS increased significantly in the controls compared to the normal dogs. LD RSV therapy tended to increase the protein expression of eNOS which was restored to near-normal values by HD RSV. Treatment with both LD RSV and HD RSV decreased the protein expression of nNOS and iNOS, but the decrease did not reach statistical significance.

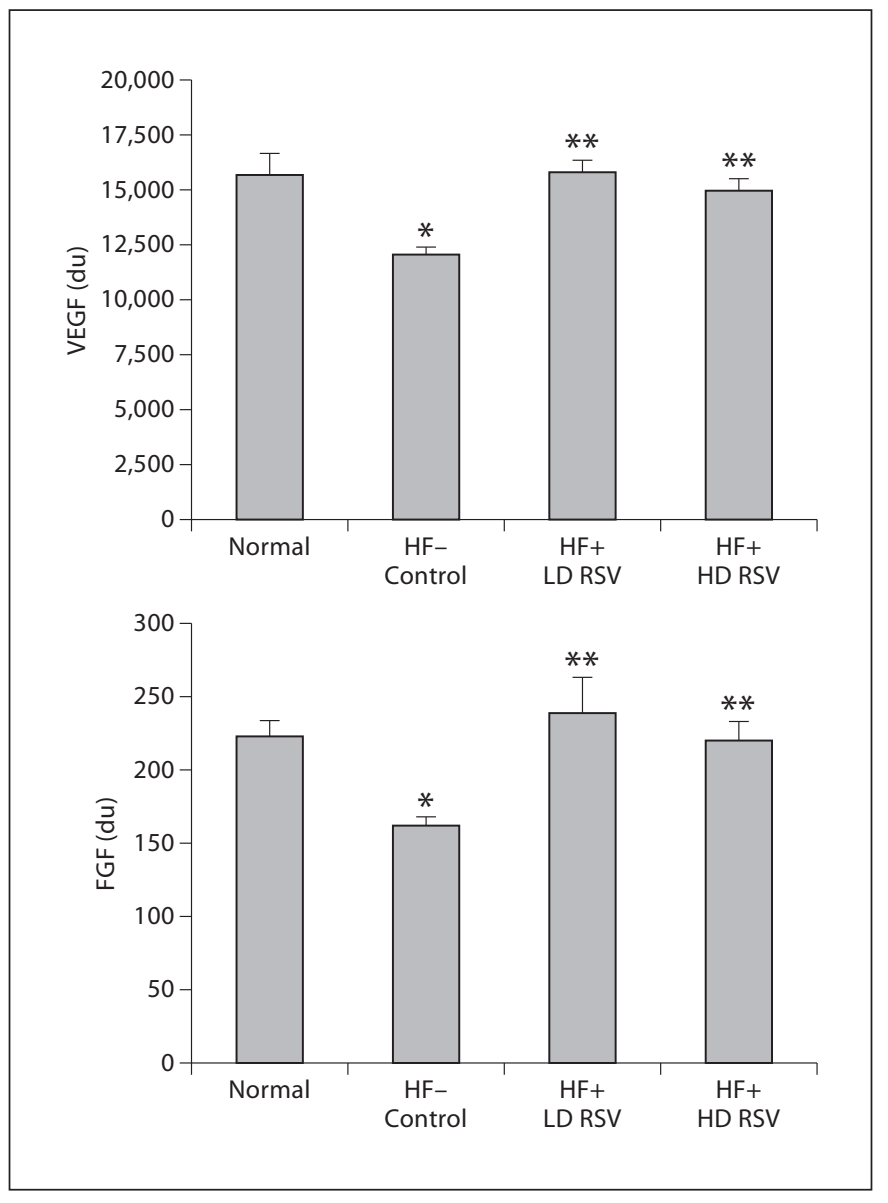

Fig. 3. VEGF and FGF protein levels in densitometric units (du) in normal dogs, HF control dogs, HF dogs randomized to LD RSV and HF dogs randomized to HD RSV. ${ }^{*} \mathrm{p}<0.05$ vs. Normal; ** $\mathrm{p}<0.05$ vs. Control.

\section{Discussion}

The use of statins in the clinical setting of HF has always been a matter of debate, beginning with the early pathophysiological controversy over their potentially harmful and potentially beneficial biological effects [15], and reaching its climax in the post-CORONA (Controlled Rosuvastatin Multinational Study in Heart Failure) and post-GISSI-HF (Gruppo Italiano per lo Studio della Sopravvivenza nell'Infarto Miocardico Heart Failure trial) era [7, 8]. Overall, randomized controlled trials of RSV, simvastatin and atorvastatin in patients with HF have shown that statin therapy, although exerting neutral effects on cardiovascular and noncardiovascular mortality, ameliorates LV systolic function and reduces hospitalizations for worsening HF [9]. These positive effects 
may translate into significant benefits in terms of patients' quality of life and of the socioeconomic impact of the syndrome. In the CORONA trial, therapy with RSV in patients with mild to moderate HF showed positive effects on the primary end points [7]. The trial also demonstrated an overall reduction in hospitalization [7]. More recent evidence with higher doses of RSV ( $40 \mathrm{mg}$ vs. the $10 \mathrm{mg}$ used in the CORONA and GISSI-HF trials), comparable to that used in our studies, is particularly encouraging and suggestive of the more extensive antiremodeling and endothelial function- enhancing effects of more aggressive dosages [10]. It appears legitimate, therefore, to further explore the mechanisms potentially underlying the beneficial effects of these agents on the failing LV.

Earlier studies from our group showed that HD RSV prevents progressive LV dysfunction and remodeling in normocholesterolemic dogs with moderate HF via nonlipid-lowering beneficial effects [3]. Our study demonstrates that HD RSV exerts these beneficial effects on global and structural LV remodeling via the modulation of several molecular targets including Nab1, PTEN, PI3K, mTOR, IL6, cKit, Sca1, VEGF, FGF, eNOS, iNOS and nNOS. These genes are, in turn, mediators of different signaling pathways involved in the regulation of adaptive and maladaptive hypertrophy [16, 17], inflammation [18], BMSC mobilization, migration and differentiation $[5,6$, 19] as well as cardiac and vascular function [20]. Taken together, these data do support the hypothesis that RSV possesses pleiotropic properties, which would account for the beneficial effects observed in our studies.

Maladaptive cardiac myocyte hypertrophy is one of the key features of the failing heart [21]. Statin therapy has been associated with antihypertrophic effects in experimental models of HF, likely related to the inhibition of the isoprenylation of small G-proteins, mainly Rac $[22,23]$. We previously showed that both LD RSV and HD RSV are associated with a significant reduction of myocardial cross-sectional area, which is a measure of cardiomyocyte hypertrophy [3]. In this study, therapy with RSV was associated with a favorable modulation of molecular signaling pathways acting upstream of small G-proteins. Nab1 inhibits cardiomyocyte hypertrophy via the repression of the transcription factor Egr and is upregulated in conditions of pathological cardiac hypertrophy including experimental and human HF [24]. We observed that HD RSV induced a significant modulation of Nab1 overexpression down to levels similar to that seen in normal dogs. The PI3K/PTEN signaling is involved in the regulation of several cellular responses to physiological and pathological stimuli acting upstream of several effectors including Akt and mTOR [16, 17, 25]. Cardiovascular effects of the PI3K/PTEN axis include the modulation of cell survival and apoptosis, myocyte hypertrophy, myocardial contractility and electrophysiological properties, energy metabolism and mechanotransduction and coronary angiogenesis $[16,17]$. PI3K activity has an essential role in basal cell growth and in adaptive (physiological) and maladaptive (pathological) hypertrophy $[16,17]$. PI3K is activated in many forms of pathological hypertrophy, cardiomyopathy and advanced human HF [26, 27], with Akt and mTOR likely being key mediators of such maladaption. In our study, HD RSV restored near-normal levels of both PI3K and mTOR mRNA and significantly reduced the protein expression of both genes. This suggests the PI3K/PTEN signaling pathway as a potentially novel molecular target for RSV in HF treatment. Selective inhibition of mTOR also has antiremodeling effects [28] which may be attained with RSV therapy.

It has been consistently shown that statins reduce inflammatory cytokines in experimental and human HF, potentially counteracting the deleterious effects of inflammation on HF pathogenesis and progression [2, 2931]. In the CORONA trial, median levels of high-sensitivity C-reactive protein were reduced by $31.6 \%$ in the RSV group and increased by $5.5 \%$ in the placebo group with an absolute difference of $37.1 \%(\mathrm{p}<0.001)$ [7]. In this study, HD RSV significantly decreased the HF-induced upregulation of IL-6. In a previous study, we showed that HD RSV also reduced the expression of TNF- $\alpha$ [3]. These cytokines are upregulated in HF and promote cardiomyocyte hypertrophy, apoptosis, reinduction of the fetal gene program and endothelial dysfunction, ultimately leading to worsening LV dysfunction and remodeling and reduced functional capacity [18]. The reduction of serum markers of inflammation such as TNF- $\alpha$ and IL-6 by means of statin treatment has been largely associated with improved LV EF and attenuated LV remodeling [29].

Myocardial regeneration has been and still is regarded as a potential option to support the failing heart. Evidence is available that suggests statins are capable of promoting angiogenesis via the activation of the PI3K/Akt signaling pathway [4]. Accordingly, statin therapy may result in increased numbers of endothelial progenitor cells (EPCs), induction of EPC differentiation, improved EPC survival and VEGF-induced migration and myocardial colonization $[4,5]$. In our study, RSV concomitantly upregulated the expression of stem cell markers and of VEGF and FGF, all of which possibly contribute to the observation of an increased capillary density, a finding 
potentially consistent with drug-induced myocardial neovascularization [3]. A limitation of this study is the absence of direct histological and/or biochemical studies in heart, tissue to support a potential causal association between the upregulation of growth factors and the development of neovascularization, and between the upregulation of stem cell markers and BMSC mobilization, migration and differentiation.

Lastly, we observed a near normalization of the expression of NOS isoforms associated with HD RSV therapy. nNOS and eNOS are constitutively present in the heart, while iNOS is expressed in disease states in response to inflammatory stimuli [20]. NOS isoforms contribute to the control of many cardiac and vascular functions and a perturbation of their balance is seen in cardiac disease. Disregulation of NO and increased oxidative and nitrosative stress are implicated in the genesis of $\mathrm{HF}$ $[32,33]$. Inflammatory cytokine-induced downregulation of eNOS expression seems to play a major role in the genesis of endothelial dysfunction in HF. Supportive evidence indicates how statin therapy promotes the recovery of endothelial function through increased eNOS expression and reduced oxidative stress $[34,35]$. Nonetheless, in an experimental model of myocardial infarction in wildtype and eNOS-deficient mice, increased eNOS availability was an essential requirement for the statin-induced improvement of EPC mobilization, myocardial neovascularization, LV dysfunction, interstitial fibrosis and survival [36].

Despite evidence in support of a biological rationale for the use of statins in HF in our translational animal model, large randomized clinical trials of RSV in patients with HF failed to show a survival benefit conferred by statin therapy. A major difference between the animal model and the clinical trials is the issue of optimal back- ground medical therapy which was in place in clinical trial patients but not in animal studies. In addition, the study in animals focused primarily on LV functional improvements resulting from RSV therapy, whereas large clinical trials focused primarily on mortality. A recent meta-analysis of randomized control trials of statins versus placebo in patients with HF clearly showed that statins improve LV EF and decrease hospitalization for worsening HF [9]. Although LV EF data are not available from the CORONA and GISSI-HF trials, data from smaller clinical studies with HD RSV in patients with HF are in line with findings in our translational animal model of HF. A recent study by Erbs et al. [10] reported a significant increase in LV EF driven primarily by a significant decrease in LV end-systolic volume in patients with systolic HF randomized to a 3-month therapy with $40 \mathrm{mg}$ RSV compared to placebo. These findings are also in line with those of our animal model [3].

In conclusion, the results of this study are suggestive of a pleiotropic effect of RSV, especially at higher doses, in the treatment of dogs with moderate HF. RSV pleiotropic properties include antihypertrophic, anti-inflammatory, BMSC mobilizing and proangiogenetic/myocardial regenerative effects elicited by the modulation of the expression of several molecular targets. Modulation of the $\mathrm{PI} 3 \mathrm{~K} / \mathrm{PTEN} / \mathrm{Akt}$ signaling pathway is a key mediator of the beneficial effects observed with chronic HD RSV monotherapy.

\section{Acknowledgements}

This study was supported, in part, by research grants from AstraZeneca US and the National Heart, Lung, and Blood Institute PO1 HL074237-07.

\section{References}

1 Baigent C, Keech A, Kearney PM, et al, Cholesterol Treatment Trialists' Collaborators: Efficacy and safety of cholesterol-lowering treatment: prospective meta-analysis of data from 90,056 participants in 14 randomised trials of statins. Lancet 2005;366:1267-1278.

-2 Ramasubbu K, Estep J, White DL, Deswal A, Mann DL: Experimental and clinical basis for the use of statins in patients with ischemic and nonischemic cardiomyopathy. J Am Coll Cardiol 2008;51:415-426.
- 3 Zacà V, Rastogi S, Imai M, et al: Chronic monotherapy with rosuvastatin prevents progressive left ventricular dysfunction and remodeling in dogs with heart failure. J Am Coll Cardiol 2007;50:551-557.

4 Kureishi Y, Luo Z, Shiojima I, et al: The HMG-CoA reductase inhibitor simvastatin activates the protein kinase Akt and promotes angiogenesis in normocholesterolemic animals. Nat Med 2000;6:1004-1010.
5 Dimmeler S, Aicher A, Vasa M, et al: HMGCoA reductase inhibitors (statins) increase endothelial progenitor cells via the PI 3-kinase/Akt pathway. J Clin Invest 2001;108: 391-397.

-6 Llevadot J, Murasawa S, Kureishi Y, et al: HMG-CoA reductase inhibitor mobilizes bone marrow-derived endothelial progenitor cells. J Clin Invest 2001;108:399-405.

7 Kjekshus J, Apetrei E, Barrios V, et al: Rosuvastatin in older patients with systolic heart failure. N Engl J Med 2007;357:2248-2261. 
8 Tavazzi L, Maggioni AP, Marchioli R, et al: Effect of rosuvastatin in patients with chronic heart failure (the GISSI-HF trial): a randomised, double-blind, placebo-controlled trial. Lancet 2008;372:1231-1239.

-9 Lipinski MJ, Cauthen CA, Biondi-Zoccai GG, et al: Meta-analysis of randomized controlled trials of statins versus placebo in patients with heart failure. Am J Cardiol 2009; 10:1708-1716.

10 Erbs S, Beck EB, Linke A, et al: High-dose rosuvastatin in chronic heart failure promotes vasculogenesis, corrects endothelial function, and improves cardiac remodeling - results from a randomized, doubleblind, and placebo-controlled study. Int J Cardiol 2011;146:56-63.

-11 Sabbah HN, Stein PD, Kono T, et al: A canine model of chronic heart failure produced by multiple sequential intracoronary microembolization. Am J Physiol 1991;260:H1379H1384.

12 No authors listed. Position of the American Heart Association on Research Animal Use. Circulation 1985;71:849A-850A.

13 Rastogi S, Imai M, Sharov VG, Mishra S, Sabbah HN: Darbepoetin-alpha prevents progressive left ventricular dysfunction and remodeling in nonanemic dogs with heart failure. Am J Physiol Heart Circ Physiol 2008; 295:H2475-H2482.

14 Rastogi S, Sharov VG, Mishra S, et al: Ranolazine combined with enalapril or metoprolol prevents progressive LV dysfunction and remodeling in dogs with moderate heart failure. Am J Physiol Heart Circ Physiol 2008; 295:H2149-H2155.

15 van der Harst P, Voors AA, van Gilst WH, Böhm M, van Veldhuisen DJ: Statins in the treatment of chronic heart failure: biological and clinical considerations. Cardiovasc Res 2006;71:443-454.
16 Oudit GY, Sun H, Kerfant BG, Crackower MA, Penninger JM, Backx PH: The role of phosphoinositide- 3 kinase and PTEN in cardiovascular physiology and disease. J Mol Cell Cardiol 2004;37:449-471.

17 Oudit GY, Penninger JM: Cardiac regulation by phosphoinositide 3-kinases and PTEN. Cardiovasc Res 2009;82:250-260.

18 Mann DL: Inflammatory mediators and the failing heart: past, present, and the foreseeable future. Circ Res 2002;91:988-998.

19 Asahara T, Masuda H, Takahashi T, et al: Bone marrow origin of endothelial progenitor cells responsible for postnatal vasculogenesis in physiological and pathological neovascularization. Circ Res 1999;85:221228.

20 Umar S, van der Laarse A: Nitric oxide and nitric oxide synthase isoforms in the normal, hypertrophic, and failing heart. Mol Cell Biochem 2010;333:191-201.

21 Jessup M, Brozena S: Heart failure. N Engl J Med 2003;348:2007-2018

22 Liao JK: Statin therapy for cardiac hypertrophy and heart failure. J Investig Med 2004; 52:248-253.

23 Laufs U, Kilter H, Konkol C, Wassmann S, Bohm M, Nickenig G: Impact of HMG CoA reductase inhibition on small GTPases in the heart. Cardiovasc Res 2002;53:911-920.

24 Buitrago M, Lorenz K, Maass AH, et al: The transcriptional repressor Nab1 is a specific regulator of pathological cardiac hypertrophy. Nat Med 2005;11:837-844.

25 Sugden PH: Ras, Akt, and mechanotransduction in the cardiac myocyte. Circ Res 2003;93:1179-1192.

26 Baba HA, Stypmann J, Grabellus F, et al: Dynamic regulation of MEK/Erks and Akt/ GSK-3beta in human end-stage heart failure after left ventricular mechanical support: myocardial mechanotransduction-sensitivity as a possible molecular mechanism. Cardiovasc Res 2003;59:390-399.

27 Haq S, Choukroun G, Lim H, et al: Differential activation of signal transduction pathways in human hearts with hypertrophy versus advanced heart failure. Circulation 2001; 103:670-677.
28 Buss SJ, Muenz S, Riffel JH, et al: Beneficial effects of mammalian target of rapamycin inhibition on left ventricular remodeling after myocardial infarction. J Am Coll Cardiol 2009;54:2435-2446.

29 Sola S, Mir MQS, Lerakis S, Tandon N, Khan BV: Atorvastatin improves left ventricular systolic function and serum markers of inflammation in nonischemic heart failure. J Am Coll Cardiol 2006;47:332-337.

30 Tousoulis D, Antoniades C, Bosinaku E, et al: Effects of atorvastatin on reactive hyperemia and inflammatory process in patients with congestive heart failure. Atherosclerosis $2005 ; 178: 359-363$.

- 31 Mozaffarian D, Minami E, Letterer RA, Lawler RL, McDonald GB, Levy WC: The effects of atorvastatin $(10 \mathrm{mg})$ on systemic inflammation in heart failure. Am J Cardiol 2005;96:1699-1704.

32 Pacher P, Schulz R, Liaudet L, et al: Nitrosative stress and pharmacological modulation of heart failure. Trends Pharmacol Sci 2005; 26:302-310.

33 Takimoto E, Kass DA: Role of oxidative stress in cardiac hypertrophy and remodeling. Hypertension 2007;49:241-248.

34 Ferrari R, Agnoletti L, Comini L, et al: Oxidative stress during myocardial ischaemia and heart failure. Eur Heart J 1998;19(suppl B):B2-B11.

35 Lefer AM, Scalia R, Lefer DJ: Vascular effects of HMG CoA-reductase inhibitors (statins) unrelated to cholesterol lowering: new concepts for cardiovascular disease. Cardiovasc Res 2001;49:281-287.

- 36 Landmesser U, Engberding N, Bahlmann $\mathrm{FH}$, et al: Statin-induced improvement of endothelial progenitor cell mobilization, myocardial neovascularization, left ventricular function, and survival after experimental myocardial infarction requires endothelial nitric oxide synthase. Circulation 2004;110: 1933-1939. 